\title{
A Smart Retrofitted Meter for Developing Countries
}

\author{
Eliel Keelson \\ Computer Engineering Dept., \\ Kwame Nkrumah University of \\ Science and Technology, \\ Kumasi, Ghana
}

\author{
K. O. Boateng \\ Computer Engineering Dept., \\ Kwame Nkrumah University of \\ Science and Technology, \\ Kumasi, Ghana
}

\author{
Isaac Ghansah \\ Computer Science Dept., \\ California State University, \\ Sacramento, \\ U.S.A
}

\begin{abstract}
Modern trends of managing energy consumption have proved the smart metering system as an effective scheme. With its capability of transmitting energy usage information in realtime to parties that matter, such as power generation stations, transmission stations, distribution stations and customers, more qualitative decisions concerning generation, distribution and usage of power are made. This energy usage information helps to match demand with supply hence reducing cost of production, increasing profitability, reducing waste and higher customer satisfaction. Despite the many benefits offered by smart meters, most developing countries in Africa such as Ghana still hold on to standalone energy meters that offer less capabilities. This paper brings to light some important drawbacks of smart meters implemented in other parts of the world and suggest possible solutions. The paper also assays the possibility of implementing secured smart retrofitted electric energy meters in developing countries in Africa taking the suggested solutions into consideration. This implementation does not require the replacement of the old meters.
\end{abstract}

\section{General Terms}

Embedded Systems, Smart Metering Systems

\section{Keywords}

Smart meter, retrofitted, GSM, Africa, developing countries

\section{INTRODUCTION}

The world's demand for electric energy has been on a constant surge [1]. It has been forecast that this demand would soar up by a percent or two each year [2]. This increase in demand is mainly due to the increase in population and economic development. Energy Information Administration (EIA) predicts that a greater portion of the global demand of energy would emerge from developing countries (of which Ghana and most African countries are a part) [3]. Bearing in mind that the unique properties of electricity generated in bulk make it impossible to be stored for future use, it is important then to match its consumption with generation [4].

Currently the cost of generation, transmission and distribution is solely dependent on consumption [2]. So if more than what is needed within a particular time frame is generated, it is up to the consumer to pay for what was used as well as what was wasted. The environment is also affected through the emission of carbon dioxides which are usually produced during generation of electric energy; whether from a renewable or non-renewable source.

The high demand for electric energy has thrust some developing countries into energy crisis. For example, Ghana's Volta River Authority (main power generating company), on several occasions has had to embark on load shedding exercises in order to partly meet the growing needs of its consumers [5]. Load shedding stifles business growth and development, which directly has a negative impact on the economy.

There is an immediate need to properly manage the growing demand of electric energy. An effective way of doing this is by matching demand with supply, thus reducing wasted power, as well as reducing the demand of peak power. The smart meter offers both the supply-side and demand-side the ability to achieve this goal.

In this paper some important drawbacks of smart meters, implemented in other parts of the world, are highlighted and solutions to them are suggested. Design features and a block diagram for a smart retrofitted meter for developing countries in Africa are presented. The features serve to correct the drawbacks or reduce their effects. The proposed design is based on the reuse of old standalone energy meters hence it is cost saving.

In section 2 of this paper, a brief explanation of what smart meters are and some of their benefits are stated. In section 3 some drawbacks are presented. Section 4 presents suggested measures to mitigate those drawbacks. Section 5 presents the features and block diagram of the proposed smart meter targeted for developing countries. Section 6 presents a prototype design with simulation results. Finally a conclusion is drawn in section 7 .

\section{SMART METERS}

A Smart Meter can be described as an advanced metering device that usually records consumption of electrical energy in intervals of an hour or less and transmits the recorded information over a communication medium [6]. It is often considered as the basic unit of most smart grids [7].

Smart meters were introduced by utility companies in the year 2009 with the primary objective to match energy consumption with generation [8]. This is made possible by the continual provision of real-time or near real-time energy consumption information by smart meters to utility companies. Equipped with this information, utility companies are able to assess how much energy has been consumed over a particular time interval and estimate how much energy is to be generated to cover a particular time frame.

There are a number of benefits that smart meters offer to both utilities and consumers. Some of these are:

1. Real-time energy consumption information helps consumers to adjust their consumption lifestyle leading to energy conservation and lower bills.

2. Remote and accurate meter reading and billing thus bringing an end to estimated bills, reducing the cost for meter reading and increasing privacy. [9]

3. Remote connection and disconnection. 
4. Utilities are better informed hence can plan to meet the needs of the consumer - higher satisfaction.

5. Remote detection of power outages by utilities rapid recovery.

6. Power outage notifications to consumers.

7. Detection of energy theft (illegal connections). [10]

For the above reasons and more, many countries such as Botswana, Canada, Italy, Netherlands, New Zealand, the Nordic Countries, South Africa, Spain, United Kingdom and United States of America have taken the initiative to replace most of their energy meters with smart meters [11][12]. In the United States for example, as of June 2011, about 20 million smart meters were installed and it is estimated that by 2015 these numbers would reach 65 million, representing half the population of US households [13].

To achieve the above stated reasons, a lot of work has been done in this area to develop reliable standards for smart metering and smart grid applications. One widely used standard is the Open Smart Grid Protocol (OSGP) issued by the European Telecommunications Standard Institute (ETSI) [14].

\section{DRAWBACKS OF SMART METERS}

Despite the many benefits that smart meters present there have been a few drawbacks; most of them were gathered from the complaints of consumers who use smart meters. Some of the observed drawbacks have to do with, among other things, the issue of

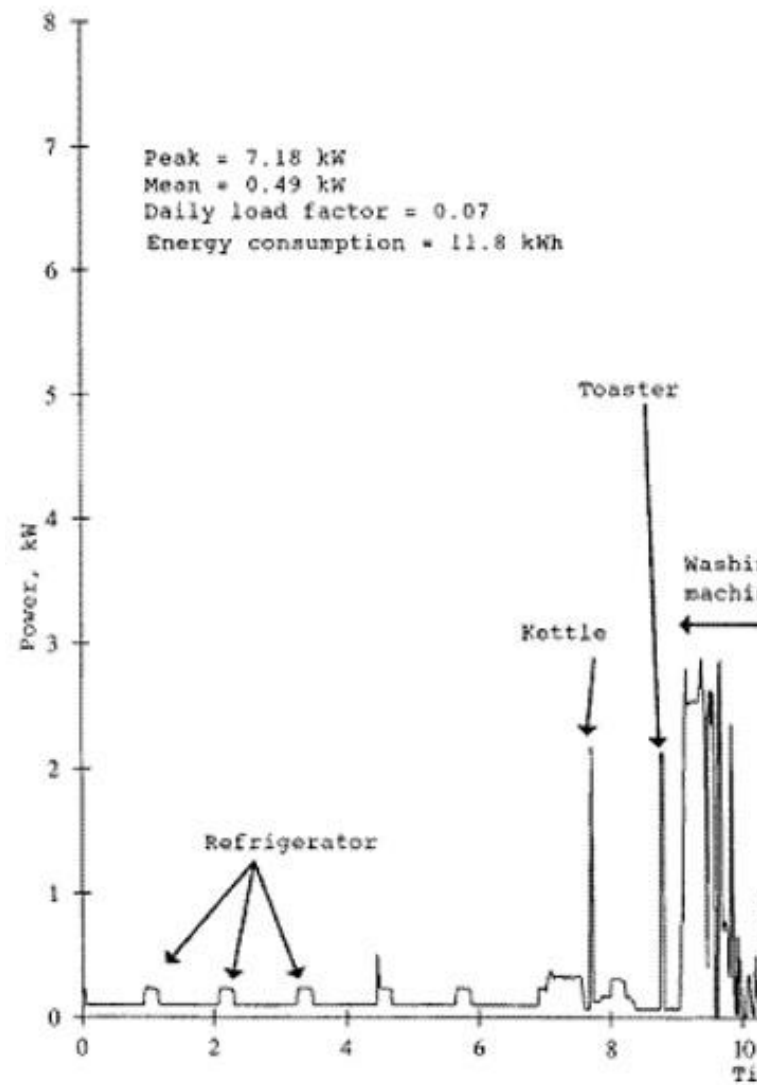

Fig 1: Sample data from a smart meter showing various gadgets' energy consumption [15] [16].

Consumers are not even certain if the information transmitted can be read by other unauthorized parties. There is a wide
1. Privacy and Security.
2. High cost of deployment.
3. Little benefits to consumers.

Because of these drawbacks some countries, states or regions have requested the ban of smart meters. For example, the attorney generals of both Illinois and Connecticut have stated that they do not believe smart meters provide any benefit to consumers, as a result most consumers in those states have demonstrated against the installation of smart meters [12][15].

In the next three subsections the paper would throw more light on the above stated drawbacks.

\subsection{Privacy and Security}

There have been a number of reports by consumers that smart meters are being used by utility companies as a tool to spy on their liberty. They often explain that since these intelligent devices transmit consumer's energy usage information at regular intervals, the parties that receive such information are capable of discerning from the meter's consumption pattern whether the consumer is at home or not; or even trace what kind of electrical gadgets the consumer uses from time to time. Every electrical gadget has a unique energy fingerprint hence it is easy for one to guess from the data gathered which gadgets a consumer has, what times of the day they are used, how long and often he/she uses them [15]. For example, from figure 1 below one can tell the various gadgets that the consumer used within the day and the duration of use. It can also be inferred from the graph that the consumer loves tea.
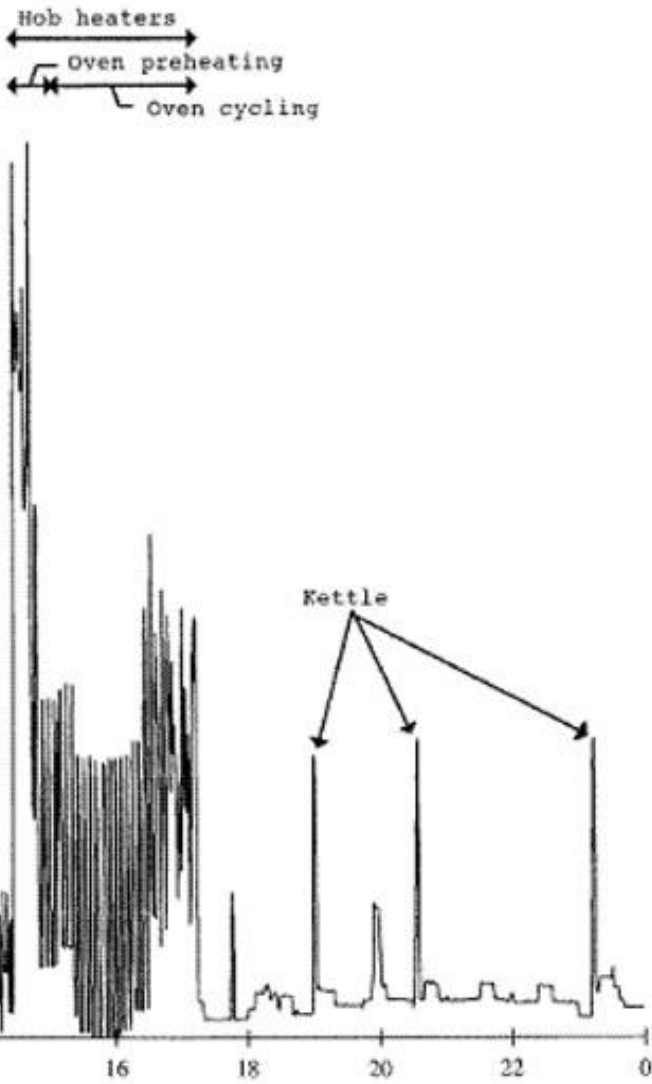

range of people/organizations that would do anything to have access to this information. For example, marketers would 
need this information to be able to profile consumers for particular advertisements, the police would need such information to fish out suspicious activities and burglars may need such information to know whether their victims are at home or not as well as tell the various gadgets they are to look out for [16]. This is an intrusion of the privacy of consumers and a threat to their security.

\subsection{High Cost of Deployment}

In some developed countries where there have been a massive roll-out of smart meters; thus replacing the old standalone energy meters, there have been high financial costs to bear. In most of these countries a majority of the cost has been absorbed by the government, while in other countries consumers and other private power agencies are made to bear the cost. In July 2010, the Department of Energy and Climate Change (DECC) of the United Kingdom Impact Assessment estimated the accelerated rollout to cost around $£ 10$ billion [12]. Also in February 2011, Connecticut Light and Power estimated that taxpayers would have to generate $\$ 500$ million for the provision and replacement of the old meters with smart meters [15]. It is hard to say whether all the old meters they are replacing can serve any future purpose or are completely rendered unusable.

\subsection{Little Benefits}

Some consumers have complained that a vast majority of the benefits that smart meters offer are for utilities. The consumer is only given the capability of reading his/her power consumption information whereas the retailers and electricity generation companies enjoy many of the other benefits such as remote meter reading, remote disconnection and reconnection and remote update of tariffs, among others. Some consumers are not satisfied with the benefits that are presented to them by these meters, especially considering the large sums of taxpayers' money that went into the provision of these meters [15]. Also some are of the view that there is little or no significant reduction in demand for peak power after the installation of smart meters - the demand still remains the same hence not achieving one of the primary objectives of smart meters [12].

One is tempted to say that the drawbacks stated above, especially the issue of high cost of rolling out, may be reasons why some developing countries in Africa, like Ghana, have not yet taken the initiative of replacing their standalone energy meters with smart meters. In the following section the paper attempts to suggest measures that developing countries in Africa can apply to lessen the effect of these drawbacks in order to achieve the full benefits of smart meters. The paper would then propose a secured smart retrofitted meter that can be implemented in these countries.

\section{MEASURES TO MITIGATE DRAWBACKS}

In dealing with the issue of security and privacy the following points are worth noting:

1. All smart meters should be tamper-proof or have some way of telling if they have been tampered with.

2. Communication should only be between designated firewalled Meter Data Management Systems (MDMS) and tamper-proof meters. No communication should take place if meters have been tampered with.

3. Every meter/ MDMS should be authenticated before communication takes place.
4. All data exchanged between meter and MDMS must be encrypted using Elliptic Curve Cryptography (ECC), which the National Security Agency (NSA) in 2005 declared as the most secure public key cryptographic system [17].

ECC is highly desirable for small devices like the smart meter, since it requires smaller key sizes as compared to the other cryptographic schemes, whiles delivering the same level of security; if not better [18].

To add to the above stated points policies should be put in place to govern how energy usage data is exchanged and who has the right of access to consumers' usage data. At best there should be separation of privileges as a security measure.

To address the issue of high cost of deployment, the paper suggests that instead of completely replacing all energy meters with smart meters, they should be retrofitted with modules that provide the desired smart meter functionality. Also these modules should be equipped with communication devices that are based on technologies popularly used in such countries. The paper proposes the use of Global System for Mobile Communications' (GSM's) connectionless Short Message Service (SMS) as the main means of communication. GSM has already taken deeper roots in most parts of Africa as compared to other wireless methods of communication. A greater population of mobile operators in Africa use GSM [19]. Also since SMS is connectionless in nature congestion would be minimized in the network. This perhaps would be a less expensive solution and should be targeted at developing countries. This would also mean that the existing meters would still have use. With this, in case a consumer requests his/her old meter back there would only be the need to deactivate/remove the added modules. The details of the components that these modules should contain would be discussed in the next section.

Finally to tackle the subject of consumer dissatisfaction of the number of benefits offered them by smart meters the paper proposes that more services be offered them. Services such as the following should be added:

1. Consumers should be sent periodic messages (either SMS or email) concerning meter status - whether power is on or off, current tariff, usage statistics and tips for energy conservation.

2. Consumers should be capable of remotely turning on or off their power via an MDMS, as another option for conserving energy even when they are far from home.

3. Consumers should also be given the option to set energy thresholds (say for a day, month or year) and receive notifications when nearing those thresholds.

4. Utilities should reward consumers who alter their consumption lifestyles to conserve energy.

These above stated points would help developing countries in Africa mitigate these drawbacks.

\section{THE PROPOSED SMART METER}

From the issues and suggestions raised in the sections above, the paper proposes a secured smart retrofitted meter with the following features.

1. An LCD for displaying usage information.

2. A relay for disconnection and reconnection. 
3. A zero crossing detector for calculating the phase angle.

4. A voltage measuring circuit for power quality measurements.

5. A real-time clock (RTC) for keeping system time and logging.

6. A GSM modem for communication.

7. A coupler for counting electricity units measured by the existing meter to help reckon consumption.

8. A tamper-proof casing or detection mechanism.

9. A microcontroller unit programmed to perform smart meter functions in conjunction with the above stated components.

Figure 2 is a block diagram showing the interconnection of components.

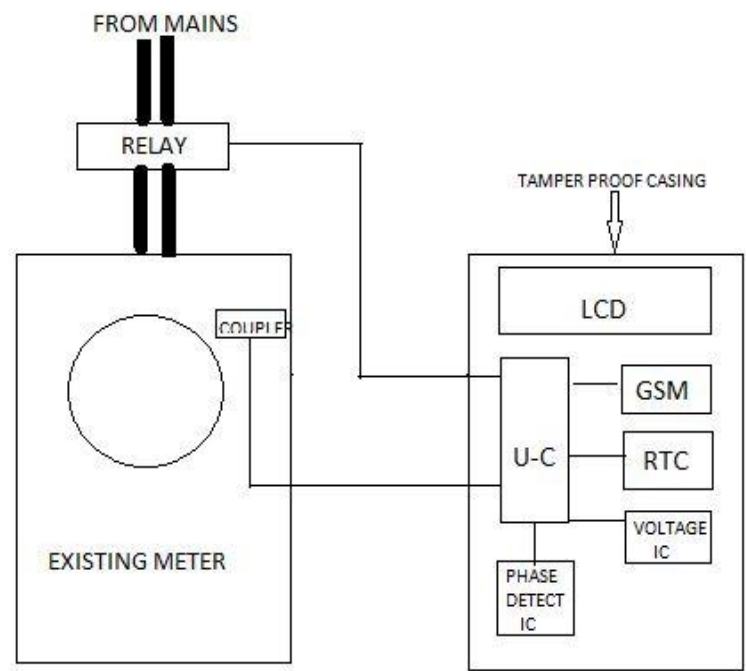

Fig. 2: A block diagram showing the interconnection of components

\section{PROTOTYPE DESIGN AND SIMULATION}

To illustrate the proposed retrofit, a circuit was designed from the block diagram and simulated. The design was used to test the feasibility of having a module that provides smart meter functionality to an existing meter. The existing energy meter used for the test has a light emitting diode (LED) which blinks each time a defined quantity of electric energy is consumed.

The retrofit was designed using a PIC18F458 microcontroller, a relay, a 20x4 alphanumeric LCD, a DS1307 RTC, an NPN optocoupler for phase detection, an ACS712 hall effect linear current based sensor for voltage measurement, a light dependent resistor (LDR) to detect LED pulses from the existing meter, a switch to detect when the case of the module is tampered with and a 5 volts battery as the main power supply for the circuit. Figure 5 shows a schematic diagram of the prototype module designed with ISIS Professional Software, release $7.9 \mathrm{SP}$ 1. The microcontroller was programmed in $\mathrm{C}$ language using CCS Compiler version 4.3.0.271.
The proposed module records energy consumption by using the LDR to detect pulses from the LED on the existing meter. The existing meter used for the test has an LED which pulsates 600 times per kilowatt hour. Each pulse from the LED is detected by the LDR which then triggers the microcontroller to count hence calculate how much energy has been consumed. The usage data is then recorded and various statistics are generated from it. Some of these statistics include the consumption per previous hour/day/month/year as well as the average consumption per hour/day/month/year. The results of these statistics are displayed in turns on the LCD as shown in figure 3.

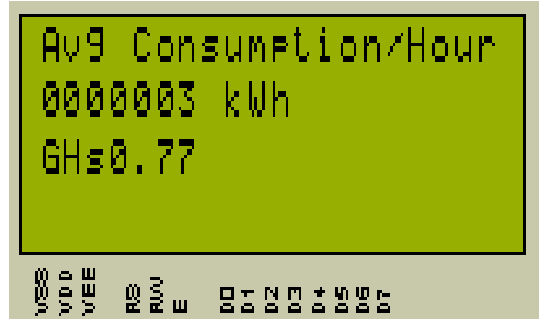

Fig. 3: The LCD displaying the average consumption per hour.

The module, at regular intervals, calculates and records various power quality measurements such as the voltage and phase angle. The results from these measurements are also displayed in turns on the LCD as shown in figure 4.

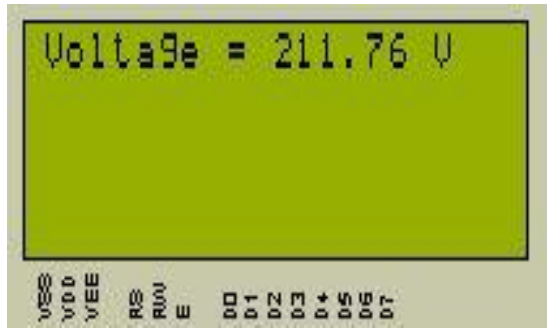

Fig. 4: The LCD displaying the measured supply voltage.

After every 20 minutes, the module transmits all recorded data together with information about current status of the meter; power on or off, connected or disconnected. All these are sent as an ECC-encrypted short message service (SMS), to the utility company via a GSM modem embedded in the module. This data could also be sent upon request of the utility company or during special events such as unfavourable power characteristics or meters being tampered with - which requires immediate action from the utility and also at the close of a day.

Disconnection/reconnection of power is done remotely when an encrypted SMS is received from the utility company requesting this service. This message could also be sent by the utility company on behalf of the consumer. When this service is requested the module sends a signal from the microcontroller unit to the relay to carry out the specified action.

The module allows different energy thresholds to be set for different periods of time, say a day, month or year. When the set threshold is reached the smart meter displays a message on the LCD as well as notifies the utility/consumer by sending an encrypted SMS. These notifications help consumers know how long they take to reach their set thresholds hence adjust their consumption lifestyle to conserve energy. 
Other notifications from the utility, such as outage/maintenance notifications and tariff updates can be sent as an encrypted SMS to the module. These notifications are displayed on the LCD until the notification expires.

The simulations proved that the proposed secured smart retrofitted meter is feasible. It provides utility companies with the necessary information needed to match demand with supply. It also provides the consumer with various usage statistics to help conserve energy. Table 1 shows a summary of the functions of the simulated retrofit.

\section{Table 1. Summary of Functions}

\begin{tabular}{|cl|c|}
\hline \multicolumn{2}{|c|}{ Description } & Verified \\
\hline 1. & Two way communication via GSM & $\checkmark$ \\
\hline 2. & $\begin{array}{l}\text { Secured transmission of usage data by } \\
\text { ECC encrypted SMS }\end{array}$ & $\checkmark$ \\
\hline 3. & Display of usage data statistics & $\checkmark$ \\
\hline 4. & Remote (dis)connection & $\checkmark$ \\
\hline 5. & Tamper proof mechanism & $\checkmark$ \\
\hline 6. & Power quality measurements & $\checkmark$ \\
\hline 7. & Setting of thresholds & $\checkmark$ \\
\hline 8. & Outage notifications and tariff updates & $\checkmark$ \\
\hline
\end{tabular}

\section{CONCLUSION}

This paper proposes a secured smart retrofitted energy meter, based on GSM technology, for developing countries especially in Africa. The proposed smart meter has features that mitigate the issues of security and privacy risks, high cost of deployment and little benefit from the consumer's perspective. The proposed smart meter was then designed and simulated. Simulation results show that the retrofit can serve as a model smart meter for future development. In future work the research team plans on working at prototype implementation and experimental deployment.

\section{ACKNOWLEDGMENTS}

Thanks to Michael Wilson, Hubert Attah and Fabrice Jean Boyoro who assisted this research. 


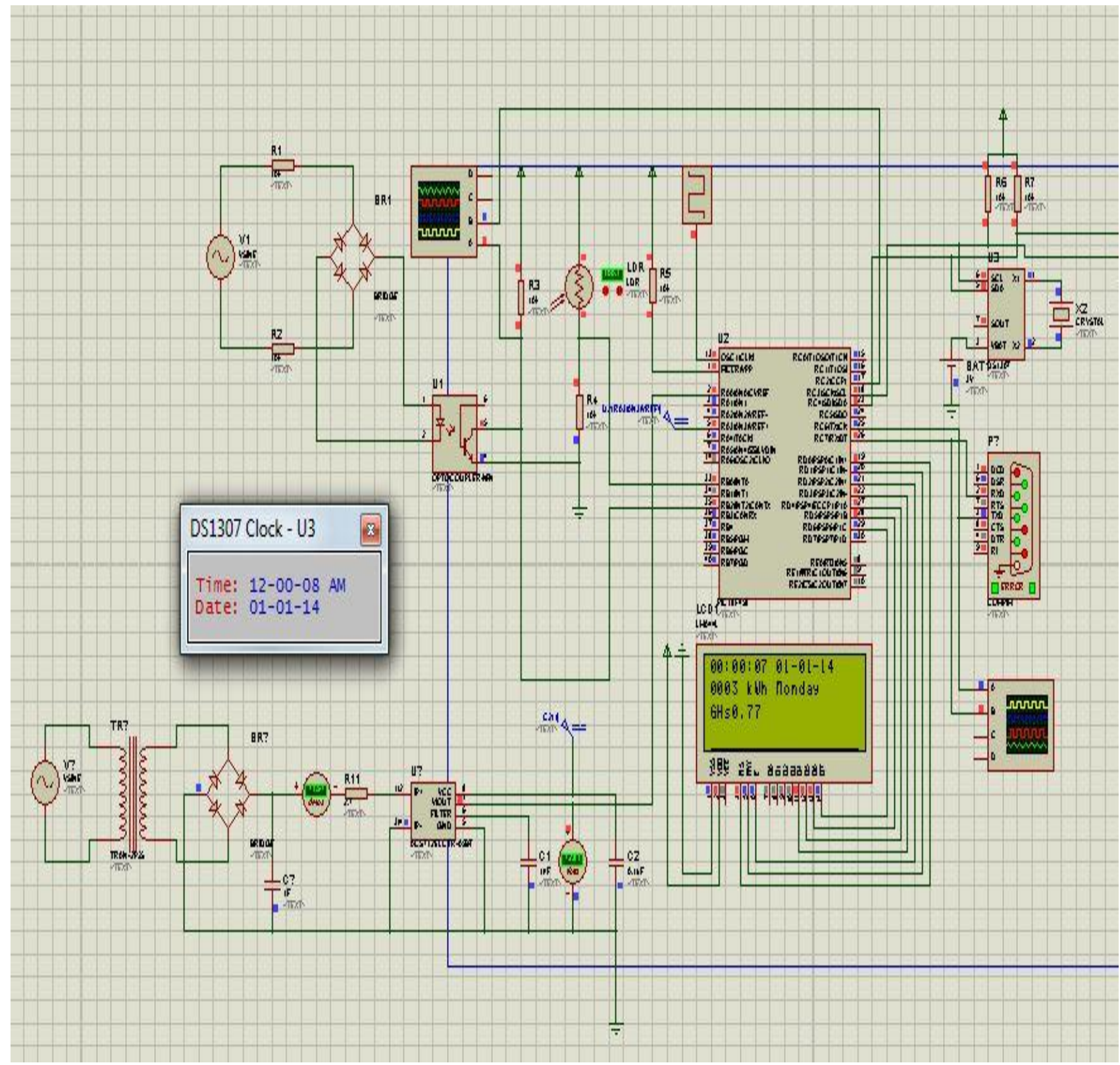

Fig. 5: A Schematic Diagram of the Simulated Circuit

\section{REFERENCES}

[1] "World Energy Consumption", Wikipedia, https://en.wikipedia.org/wiki/World_energy_consumptio n, June 2013

[2] Parliamentary Commissioner for the Environment - Te Kaitiaki Taiao a Te Whare Pāremata, "Smart Electricity Meters - How Households and the Environment can Benefit", Netherland, June 2009.

[3] Catherine Wolfram, Orie Shelef, and Paul Gertler, "How Will Energy Demand Develop in the Developing World?" pp. 1-5, January 2012.

[4] "Environmental Impact Report for the proposed establishment of a New Coal-Fired Power Station in the Lephalale Area", Limpopo Province, March 2006.

[5] Abeeku Brew-Hammond, and Francis Kemausuor, "Energy Crisis in Ghana - Drought Technology or Policy", pp. 8-14, 2007
[6] Peter H. Sierck, Principal, Industrial Hygienist, "A Technical Paper to Clarify RF Radiation Emissions and Measurement Methodologies", pp. 4-5, December 2011.

[7] Engr. Bilal Shahid, Zubair Ahmed, Adnan Faroqi, and Rao M.Navid-ur-Rehman "Implementation of Smart System Based on Smart Grid Smart Meter and Smart Appliances", 2012 International Conference on Smart Grid Systems (ICSGS 2012), IPCSIT vol.45 ,pp.1-5, 2012

[8] Tom Wilson, BSEE, Green and Healthy Homes, "Smart Grid \& Smart Meter Architecture", Wireless Safety Summit Washington DC, Oct. 5, 2011.

[9] A Joint Project of the EEI and AEIC Meter Committees, "Smart Meters and Smart Meter Systems: A Metering Industry Perspective", An EEI-AEIC-UTC White Paper, pp. 5-10 March 2011.

[10] Vinod Vasant, Kamte,ERP Lead Consultant (SAP IS-U Competency), "Smart Metering", Mphasis Corporation, Whitepaper, pp.3, June 2012. 
[11] Ernst and Young, "Smart World, Smart Utilities", Utilities Unbundled - New perspectives on the power and utilities sector-Issue 08, pp. 4-6, May 2010.

[12] "Smart Meter", Wikipedia, http://en.wikipedia.org/wiki/Smart_meter, June 2013.

[13] Ahmad Faruqui, Doug Mitarotonda, Lisa Wood, Adam Cooper, and Judith Schwartz, "The Costs and Benefits of Smart Meters for Residential Customers", The Edison Foundation, Institute of Electric Efficiency, IEE Whitepaper, pp. 2-5, July 2011.

[14] European Telecommunications Standard Institute, "ETSI GS OSG 001”, V1.1.1, pp. 11 - 27, January 2012

[15] Nina Beety, "Analysis: Smart Meter and Smart Grid Problems", Legislative Proposal, Second Edition, December 2012.
[16] Brandon J. Murrill, Edward C. Liu, and Richard M. Thompson II, Legislative Attorney, "Smart Meter Data: Privacy and Cybersecurity", Congregational Research Service, pp. 3-6 February 2012.

[17] Ann Hibner Koblitz, Neal Koblitz, and Alfred Menezes, "Elliptic Curve Cryptography: the Serpentine Course of a Paradigm Shift”, Mathematics Subject Classification, pp. 1-10, October 2008

[18] Martin Leslie, "Elliptic Curve Cryptography", Advanced Combinatorics, 40575063, pp. 8-13, June 5, 2006.

[19] Andrew Till, Technology Strategist, "GSM Wireless Data", White Paper, Psion Dacom Plc, pp. 3 - 18, October, 1999. 